\title{
¿Necesitan derechos los ríos? Comparando estructuras legales para la regulación de los ríos en Nueva Zelanda, Australia y Chile
}

\section{Do rivers need rights? Comparing legal structures for river regulation in New Zealand, Australia and Chile}

\author{
Elizabeth Macpherson'“" y Erin O'Donnell“"'
}

Este artículo aborda lo que el otorgamiento de "personalidad jurídica" a los ríos puede ofrecer a las opciones institucionales para la regulación medio ambiental del agua. Se examina esta pregunta al estudiar la aproximación adoptada para el Río Whanganui en Nueva Zelanda, el cual ostenta la calidad de persona jurídica, junto con otras entidades de administración de aguas en Chile (Juntas de Vigilancia) y Australia (Titular Victoriano de Agua Ambiental), las que no otorgan personalidad jurídica directamente a sus ríos.

Palabras clave: Personalidad jurídica; derecho ambiental; derecho de aguas comparado; regulación de los ríos.
In this article we consider what giving rivers 'legal personality' might add to existing institutional options for environmental water regulation. We examine this question by studying the regulatory approach adopted for the Whanganui River in New Zealand, which is a 'legal person', alongside other river management entities in Chile (Water Monitoring Boards) and Australia (Victorian Environmental Water Holder), which do not grant legal personality to rivers.

Keywords: Legal personality; environmental law; comparative water law; river regulation

\section{Introducción}

Desde siempre, los ríos han sido valorados por sus beneficios, tanto económicos como sustentadores de vida, para los humanos. Las estruc-

\footnotetext{
* Ponencia presentada en las XIX Jornadas de Derecho y Gestión de Aguas de la P. Universidad Católica de Chile.

** Traducción realizada por Felipe Riquelme Menares, Licenciado en Derecho, Investigador y ayudante de edición del Programa de Derecho Administrativo Económico y del Centro de Derecho y Gestión de Aguas de la Pontificia Universidad Católica de Chile.

*** Lecturer, University of Canterbury, School of Law.

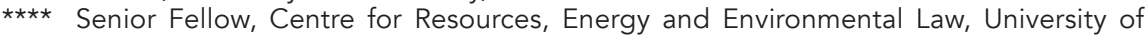
Melbourne Law School.

Recibido el 4 de diciembre de 2017 y aceptado el 25 de enero de 2018.
} 
turas legales que regulan la administración y el uso de las aguas lo han hecho teniendo en mente los siguientes principios: los recursos hídricos deben ser eficientemente administrados y la salud de estos efectivamente protegida, para efectos de mantener la calidad económica y salubre del agua, de manera que los humanos puedan seguir beneficiándose de ella. Este enfoque, conocido en el campo de la regulación de los recursos naturales como uno "antropocéntrico", hasta hace poco ha sentado las bases para las leyes de agua a través del globo.

Sin embargo, en muchas partes del mundo, podemos identificar instancias en las que los tribunales y las legislaturas comienzan a reconocer el valor de proteger los recursos naturales como un fin en sí mismos. La aplicación de un enfoque "ecocéntrico" supone que los ríos tienen un derecho intrínseco a su propia protección y mantenimiento. Al menos desde 2008, en los países sudamericanos de Ecuador y (más tarde) Bolivia, se han promulgado leyes que reconocen y protegen los "derechos de la naturaleza"1. En 2017, el Parlamento de Nueva Zelanda abrió nuevos caminos, internacionalmente, al reconocer que el río Whanganui es una "persona jurídica", con "todos los derechos, facultades, deberes y responsabilidades de una persona jurídica"2. Un enfoque similar también ha sido adoptado en India ${ }^{3}$ y Colombia ${ }^{4}$. La concesión de personalidad jurídica es la herramienta más novedosa que se ha utilizado para reconocer y proteger los derechos de la naturaleza en los ríos: es decir, que los ríos son sujetos de derechos consagrados legalmente y tienen la capacidad necesaria para demandar y ser demandados, celebrar contratos y retener propiedad a nombre propio.

Los defensores de los derechos para la naturaleza creen que el otorgar personalidad jurídica a los ríos refleja su valor inherente (no simplemente como un recurso a ser explotado) y mejora la protección de los recursos hídricos ante los posibles daños perpetrados por los seres humanos. Este cambio ecocéntrico en la gobernanza de los ríos ha sido impulsado, en gran medida, por las comunidades indígenas, que afirman sostener relaciones distintas con el agua, basadas en la tutela, la simbiosis y el respeto, las que estarían en evidente oposición al enfoque liberal y utilitarista occidental. Pero, ¿es este nuevo enfoque diferente de aquél que tienen las organizaciones existentes para efectos de resguardar y administrar los ríos? ¿Qué nuevas oportunidades ofrece el otorgamiento de personalidad jurídica para mejorar la regula-

\footnotetext{
Constitución de La República Del Ecuador 2008 (Ecuador); Constitución Política del Estado 2009 (Bolivia); Ley de Derechos de la Madre Tierra (Ley 071) 2010 (Bolivia).

2 Te Awa Tupua (Whanganui River Claims Settlement) Act 2017 (NZ).

3 Mohd. Salim con State of Uttarakhand \& others: 20 March 2017, High Court of Uttarakhand (WPPIL 126/2014) (India).

4 Centro de Estudios para la Justicia Social "Tierra Digna" y otros con el Presidente de la República y otros: 10 noviembre 2016, Corte Constitucional, Sala Sexta de Revisión (No: T-5.016.242) (Colombia).
} 
ción ambiental, en beneficio de los ríos, además de aquel cuya explotación reporta a los humanos?

Se ha considerado esta cuestión al contrastar el modelo de Nueva Zelanda con dos estructuras jurídicas comparadas que tienen directa relación con los ríos, las que, a su vez, son "personas jurídicas" pero que no otorgan dicha calidad directamente a sus propios ríos: el Titular Victoriano de Agua Ambiental en Australia (que usa su propia personalidad jurídica para actuar como una 'voz' para los ríos) y las Juntas de Vigilancia en Chile. Si bien cada uno de estos modelos existe dentro de un contexto legal, social y político distintivo, la regulación y la reforma de los marcos jurídicos de agua son muy importantes en cada país, ya que la demanda confiable y continua de suministro de agua aumenta la presión sobre los recursos hídricos.

Se argumenta que la personalidad jurídica puede ofrecer oportunidades para asegurar resultados nuevos o diferentes en la legislación y regulación ambiental en situaciones en las que el río necesite de voz propia para competir por resultados con otros interesados o usuarios. Asimismo, se cree que para que los otorgamientos de personalidad jurídica proporcionen tales oportunidades, esta debe estar acompañada por la creación de instituciones fuertes e independientes que velen por los intereses de los ríos, que se manifiesten, claramente, por los valores de los mismos, en orden de responsabilizar a los representantes y contar con derechos legalmente constituidos para usar sus aguas.

\section{Derechos de los ríos y regulación en un espectro}

La regulación ${ }^{5}$ de los recursos naturales, tanto a nivel internacional como dentro de las leyes y políticas nacionales, siempre ha estado dominada por una visión antropocéntrica ${ }^{6}$. El ecocentrismo cambia los enfoques sobre el antropocentrismo. El primero sostiene que la naturaleza tiene un valor intrínseco, no se trata solo de un recurso para ser utilizado. Por el contrario, los seres humanos, que históricamente han usado y abusado de los recursos naturales, tienen obligaciones para con la naturaleza, en orden de proteger los derechos e intereses de ella.

El ecocentrismo tiene sus raíces en movimientos de ecología profunda o verde de la década de 1960, que se alzaron en oposición al antropocentrismo y de su visión propietaria y utilitarista de la naturaleza. A principios de la década de 1970, Christopher Stone argumentó en su artículo Should Trees Standing que los derechos, consagrados a nivel legal, de los árboles, océanos, animales y del medio ambiente, en su conjunto, debían proporcionarse

\footnotetext{
5 Ver MoRgan 2007 quien sostiene que la investigación socio-legal debe combinar las perspectivas de los derechos-base (nombrando, alegando y culpando) y la regulación-base (haciendo normas, vigilando y fortalecimiento).

6 Morgera y Kulovesi 2016, 5; Donnely y Bishop 2007, 90; Magallanes 2015, 275.
} 
para "darles voz" y garantizar así su protección7. En la ley, el ecocentrismo inspiró el desarrollo de la "jurisprudencia de la Madre Tierra", que enfatiza que "la humanidad y el mundo no humano pertenecen al mismo orden moral"8 como miembros de la comunidad de la Tierra, exigiendo un cambio fundamental en la ley y en la regulación". Los defensores de "la jurisprudencia de la Madre Tierra" a menudo señalan una dicotomía entre los derechos humanos a la propiedad en los recursos naturales, versus los derechos inherentes e inalienables de la naturaleza ${ }^{10}$. Irónicamente, la jurisprudencia de la Madre Tierra ha adoptado el concepto muy humano de "derechos" para asegurar su protección ${ }^{11}$.

Aquellos que suscriben una visión ecocéntrica han sido objeto de críticas e incluso de ridículo ${ }^{12}$ en el pasado. Sin embargo, tales teorías ya no se limitan a un activismo hipotético, de base radical: han sido aplicadas y aprobadas directamente por los tribunales nacionales y las legislaturas como derechos consagrados legalmente para la naturaleza. De hecho, un reconocimiento de la necesidad de defender los derechos de la naturaleza apunta hacia una variopinta gama de protecciones ambientales para los recursos naturales y las instituciones que las hagan cumplir.

Varios casos recientes relacionados con los derechos de la naturaleza han implicado la concesión de "personalidad jurídica" a los ríos. En la mayoría de los sistemas occidentales, al menos aquellos que evolucionan fuera de la tradición del derecho romano, las "personas jurídicas" están restringidas a personas naturales (individuos), corporaciones y navíos ${ }^{13}$. Darles a los ríos personalidad jurídica tiene por intención el proporcionarles la posición que las personas y las empresas ostentan para proteger sus intereses de cara a los demás, cuando sea necesario, en los tribunales ${ }^{14}$. El primer reconocimiento legislativo de un río como una persona jurídica ocurrió en Nueva Zelanda, en marzo de 2017, cuando al río Whanganui (Te Awa Tupua) se le otorgó el estatus de persona jurídica en virtud de un acuerdo político entre el gobierno de Nueva Zelanda y el Maorí de Whanganui Iwi (tribu). La Ley Te Awa Tupua 2017 (Acuerdo de Reclamaciones del Río Whanganui) declara que el Río Whanganui es una persona jurídica, con "todos los derechos, facultades, deberes y responsabilidades de una persona jurídica"15. La legislación elabora

\footnotetext{
7 StOne 2010.

8 De Lucia 2013, 188. BerRy 1999, 4.

9 Maloney y Burdon 2014, Maloney 2016, 132; Imran, Alam y Beaumont 2014.

10 HUtChISON 2014 179; MARgIL 2017.

11 Good 2013, 34. Good sugiere que podría ser controvertido que la Tierra tenga "derecho a la vida", pero es a través de este derecho que nacen los derechos de la naturaleza.

12 ELDER 1984.

13 DaVies y Naffine 2001.

14 O'DONNELL 2017.

15 Te Awa Tupua (Whanganui River Claims Settlement) Act 2017 (NZ) s 14.
} 
un complejo modelo de gobernanza colaborativa, presidido por una entidad "guardiana" como el "rostro humano" del río llamado "Te Pou Tupua"16. Te Pou Tupua está compuesto por un representante de la Corona y otro perteneciente a los Whanganui Iwi, para actuar en interés del río y hacer valer sus derechos.

El modelo Te Awa Tupua se considera uno de los desarrollos más significativos en materia de derechos naturales, ambientales e indígenas hasta la fecha, y ha atraído gran atención de la prensa internacional y en el debate académico y político mundial ${ }^{17}$. Sin embargo, el otorgamiento de personalidad jurídica a un río ¿es una técnica diferente de aquellas que consisten en organizaciones ya existentes y que se utilizan para proteger y gestionar los ríos; organizaciones que, a su vez, suelen tener personalidad jurídica? ¿Qué implica esta posición? ¿Por qué los ríos debieran ostentar dicha calidad?

En los siguientes segmentos, se examinarán las Juntas de Vigilancia de ríos en Chile y al Titular Victoriano de Agua Ambiental en Australia. Ninguno de estos modelos reconoce expresamente que un río sea una "persona jurídica". Se considerará aquello que ha sido aportado gracias al nuevo estado del río Whanganui como una "persona jurídica", en comparación con los modelos chileno y australiano. Al hacer esto, se estudiarán las características de cada modelo, los que se sostienen, en muchas maneras, sobre su efectividad relativa: cómo la entidad usa la personalidad jurídica (ya sea para el río o para alguna otra entidad); si los valores de los ríos (ambientales o culturales) están expresamente protegidos; si el modelo opera a nivel de cuenca completa o integrada; y si la entidad posee derechos legalmente constituidos para hacer uso del agua.

\section{Un estudio comparativo de tres modelos de administración de ríos}

\subsection{Las Juntas de Vigilancia en Chile}

\section{Regulación del agua en Chile}

La regulación del agua en Chile constituye un desafío. A pesar de ser bendecidos con una precipitación promedio alta, el agua se distribuye de manera desigual en todo el país y las condiciones climáticas varían mucho desde las zonas áridas del desierto en el norte y las zonas de alta precipitación y glaciares en el sur ${ }^{18}$. Los ríos de Chile están bajo una creciente demanda por parte de sectores como la agricultura, la industria y la amenaza del cambio climá-

16 Te Awa Tupua (Whanganui River Claims Settlement) Act 2017 (NZ) s 18.

17 Indicativo de su significación, una parte de la opinión en Te Awa Tupua Act por O'DONnell y TALBOt-JONES en The Conversation atrajo más de 120,000 visitas en el espacio de tres meses. O'Donnell Y TALBOt-Jones [En línea], 2017.

18 Consejo nacional de innovación para el desarrollo, 2016: 23. 
tico ${ }^{19}$. Al igual que en Australia y Nueva Zelanda, la calidad y cantidad del agua en muchos ríos, lagos y humedales chilenos continúa deteriorándose ${ }^{20}$.

Los marcos de la legislación hídrica en Chile se caracterizan por un enfoque de mercado integrado, regulado por el Código de Aguas de 198121, lo que implica una combinación de regulación centralizada y transferencias en el mercado de derechos de aprovechamiento. En este modelo, el agua es un "bien nacional de uso público", pero los derechos de aprovechamiento son derechos de propiedad privada protegidos por la Constitución. Con frecuencia, el caso chileno se conoce como una de las aplicaciones más extremas de la lógica de mercado para la regulación del agua, donde el Gobierno Central tiene muy poco poder de regulación y donde las decisiones de distribución las toman los usuarios privados ${ }^{22}$. En realidad, las transferencias de mercado son raras en Chile, y el gobierno ha jugado un papel clave en la asignación originaria de derechos a través de la Dirección General de Aguas ${ }^{23}$.

La Constitución chilena protege el derecho a vivir en un medio ambiente libre de contaminación, y hace responsable al Estado por la preservación de la naturaleza, concediéndole a este la prerrogativa de legislar restricciones sobre el ejercicio de derechos o libertades para proteger el medio ambiente ${ }^{24}$. Sin embargo, la Dirección General de Aguas tiene potestades limitadas para intervenir en la gestión del agua y para promover la salud acuática una vez que se asignan los derechos de aprovechamiento ${ }^{25}$. Las protecciones ambientales no se incluyeron en el diseño original del Código de Aguas de $1981^{26}$ y aquellas incorporadas tardíamente, como parte de una reforma de 2005, fueron mínimas. Estas incluyen limitaciones en las extracciones de agua subterránea en ciertos humedales de las regiones del norte de Tarapacá y Antofagasta, en respuesta a la extracción excesiva de agua desde estos acuíferos $^{27}$. Las protecciones ambientales también incluyen el establecimiento de caudales ecológicos mínimos, pero solo cuando se otorgan nuevos derechos $^{28}$, gran parte del agua de Chile ya estaba asignada cuando esta limitación entró en vigencia ${ }^{29}$. Finalmente, las enmiendas al Código de Aguas, del año 2005, permiten al Presidente de la República decretar reservas de agua, en las que no se pueden otorgar nuevos derechos de aprovechamiento, in-

19 ECLAC y OECD 2016, 25, 228.

20 ECLAC y OECD 2016, 72.

21 Código de Aguas de 1981 (Chile), art. 1.

22 BAUER 2004.

23 Hearne y Donoso 2014.

24 Constitución Política de la República de Chile 1980, art. 19(8).

25 O’Donnell y Macpherson 2012. Vergara Blanco 2014,166.

26 BAUER 2004, 61.

27 Código de Aguas arts. 58, 63. Parsons Álvarez 2002, 117.

28 Código de Aguas art. 129 bis. Guiloff 2012, 126.

29 ECLAC y OECD, 2016: 25. 
cluso para la conservación ambiental ${ }^{30}$, aunque tales reservas han sido, en la práctica, controvertidas. La única otra forma potencial de proteger los intereses ambientales del agua es a través del proceso de evaluación de impacto ambiental $^{31}$, el que se ha utilizado para exigir el establecimiento de caudales ecológicos mínimos adaptados como medidas de mitigación para internalizar los efectos negativos de un proyecto, tanto en la calidad como en la cantidad del agua ${ }^{32}$. Sin embargo, el proceso de EIA ha sido criticado como un modelo débil para la protección del medio ambiente y del agua ${ }^{33}$.

No es de extrañar que, cuando el proyecto de reforma al Código de Aguas se instaló en la palestra en los últimos años, la protección del medio ambiente estaba ya asentada en la agenda política ${ }^{34}$. Un proyecto de ley de reforma al Código de Aguas se encuentra, actualmente, enterrado en varios procesos de revisión por comités ante el Senado, y sujeto a un feroz debate político que ataca el corazón mismo del modelo de distribución de los derechos de aprovechamiento de aguas. Las enmiendas propuestas prevén que los nuevos derechos de agua caducarán después de 30 años; establece la priorización del consumo humano y el saneamiento en la creación de nuevos derechos; y aborda el especial caso de especulación de los derechos ${ }^{35}$. Las reformas también incluyen disposiciones futuras para caudales de reserva y ecológicos mínimos. Sin embargo, decepcionantemente, la propuesta pasa por alto la necesidad de instituciones fuertes que hagan cumplir los ambiciosos objetivos ambientales, de salud y sociales, y descuida las estrategias de recuperación para abordar los problemas existentes causados por el otorgamiento excesivo de derechos de agua.

\subsection{Las Juntas de Vigilancia}

En ausencia de una consolidada regulación gubernamental del uso del agua en interés del medio ambiente, el sector privado ha jugado un papel importante en la regulación del agua a través de organizaciones privadas de usuarios de aguas ${ }^{36}$. Se trata de lo que a veces se denomina un enfoque de "autogobierno", las asociaciones de usuarios toman decisiones sobre la distribución de agua entre los titulares de derechos de aprovechamiento en

30 Código de Aguas, art. 147 bis. 'Deniega en parte solicitudes de derechos de aprovechamiento no consuntivos de aguas superficiales que indica, provincias de Valdivia y del Ranco, Región de los Ríos', Diario Oficial de la República de Chile, No 13.599, 1 Marzo 2010, pp. 13-17.

31 Ley 19.300, 1994.

32 GUILOFF 2012.

33 Tecklin, Bauer y Prieto 2011, 888.

34 Biblioteca Nacional del Congreso 2016, 2.1. Biblioteca del Congreso Nacional de Chile, 2017.

35 Cámara de Diputados (primer trámite legislativo), "Proyecto de Ley que Reforma el Código de Aguas" (Boletín N 7543-12).

36 Vergara Blanco 2014, 166; Rojas Calderón 2014. 
aguas superficiales específicas. En el caso de los ríos, este papel se lleva a cabo, a nivel de cuenca o semicuenca, por organizaciones conocidas como Juntas de Vigilancia (Juntas de Vigilancia de Agua) ${ }^{37}$.

Las Juntas de Vigilancia son corporaciones privadas, sin fines de lucro, en las que participan los "accionistas" de agua en la cuenca de un río. Por expreso mandato legal (...), los usuarios de agua (individuales o asociados) de una cuenca en particular se organizan como una junta, en la que reciben una participación accionaria en las aguas, proporcional a su respectiva cuota consignada en los derechos de aprovechamiento totales en la cuenca ${ }^{38}$. El Código de Aguas permite la constitución de Juntas de Vigilancia, las que después de un proceso de notificación administrativa y publicación, realizado por la Dirección General de Aguas, se convierten en "personas jurídicas"39. Por lo tanto, aunque no se ha reconocido a los ríos como personas jurídicas en Chile, las Juntas de Vigilancia se benefician de dicho estatuto jurídico.

El objetivo de una Junta de Vigilancia, establecido en el artículo 266 del Código de Aguas, es "administrar y distribuir las fuentes naturales de agua a las que tienen derecho sus miembros, utilizar y conservar la infraestructura hídrica común y llevar a cabo los demás fines prescritos por ley". Conforme a este mandato bastante amplio, las tareas de gestión del agua incluyen (sin límite) asegurar que la captación de agua se realice de manera eficiente a través de una infraestructura adecuada y otras medidas para asegurar la distribución completa y correcta de los derechos de aprovechamiento, pudiendo solicitar, a la Dirección General de Aguas, la declaración de estado de escasez y la de agotamiento hídrico, junto con la dictación de las medidas correspondientes para suspender el ejercicio de los derechos de aprovechamiento.

Las Juntas de Vigilancia tienen amplias facultades, dadas por el Código de Aguas, para monitorear y administrar los ríos bajo su jurisdicción, lo que incluye asegurar una distribución eficiente de los derechos de agua y generar medidas de protección y remedio para proteger la salud del río. Estos poderes, argumenta Vergara Blanco, van más allá del simple manejo de la distribución y del ejercicio de los derechos de aprovechamiento, ya que apuntan a la gobernanza y conservación de los ríos ${ }^{40}$.

"Las Juntas de Vigilancia tienen la facultad y el deber de gobernar y conservar no solo las aguas a las que sus miembros tienen derecho, sino

37 Código de Aguas art. 263-4. Ver, generalmente, 390-3 "asociaciones de canalistas" que son asociaciones de usuarios de canales que están a cargo de administrar la infraestructura primaria como embalses y canales de regadío, y las "comunidades de agua", que son las responsables de la infraestructura secundaria como los canales de distribución.

38 Código de Aguas, art. 268.

39 Código de Aguas, art. 263, '"Efectuada la referida publicación, la Junta de Vigilancia gozará de personalidad jurídica".

40 Vergara 2014, 166; Rojas Calderón 2014, 128. 
también los canales artificiales bajo su jurisdicción. En la práctica, esta gestión del agua significa determinar cuánta agua disponible existe en la cuenca del río, garantizar la conservación de la cuenca, determinar cómo se distribuirá el agua y resolver los conflictos antes del litigio".

La Dirección General de Aguas solo tiene la potestad para intervenir en una cuenca supervisada por una Junta de Vigilancia en circunstancias excepcionales, como por ejemplo, en el caso de asignación excesiva de derechos de aprovechamiento de aguas. Esto no implica que "el mercado" esté a cargo de regular el uso del agua en Chile, sino que la función pública de regular el ejercicio de los derechos de aprovechamiento de aguas ha sido dada (o delegada) por la Administración Estatal a los particulares. En este sentido, Rojas Calderón argumenta que las Juntas de Vigilancia, a pesar de ser corporaciones privadas, realizan una función "pública" por gestionar la distribución del agua del río y su salud a lo largo de toda la cuenca, lejos del Gobierno Central ${ }^{41}$. Sus defensores argumentan que las Juntas de Vigilancia representan la mejor práctica, una gestión descentralizada e integrada de los recursos hídricos en interés común, y el Banco Mundial ha abogado por el fortalecimiento de las asociaciones chilenas de usuarios de agua ${ }^{42}$.

No existe una protección expresa de los valores ambientales u otros relativos a ríos en el Código de Aguas. Sin embargo, muchas juntas de vigilancia ahora tienen programas ambientales y reclaman actuar en interés de la sostenibilidad del recurso ${ }^{43}$. La Junta de Vigilancia del Río Huasco, por ejemplo, tiene como misión ${ }^{44}$ :

"Administrar y distribuir las aguas superficiales y subterráneas de la cuenca del río Huasco y sus afluentes, de acuerdo con los derechos de cada usuario, protegiendo la cantidad y calidad del recurso, representando a los irrigadores ante el Estado y el sector privado, apoyando la gestión de las comunidades de agua y el desarrollo de la cuenca del río Huasco".

\subsection{El Titular Victoriano de Agua Ambiental en Australia}

\section{La regulación del agua en Australia}

Australia es el continente habitado más seco y, como en Chile, la regulación sostenible y eficiente del agua ha desafiado a los reguladores por mucho tiempo. Australia depende, en gran medida, de los principales depósitos de agua para apoyar el riego, y se enfrenta a una presión cada vez mayor

\footnotetext{
41 Rojas Calderón 2014, 130.

42 World Bank; Bauer 2015.

${ }_{43}$ Ver Fondo de Protección Ambiental Río Longaví (Junta de Vigilancia Río Longaví, http://www.juntariolongavi.cl/?p=67, visto el 9 octube 2017); Participación Ambiental (webpage, Junta de Vigilancia de la Cuenca de Río Huasco y sus Afluentes, http:// www.riohuasco.cl/category/participacion-ambiental/, accessed 9 October 2017).

${ }_{44}$ Misión y Visión (webpage, Junta de Vigilancia de la Cuenca de Rio Huasco y sus Afluentes, http://www.riohuasco.cl/mision-y-vision/, accessed 9 October 2017).
} 
sobre sus recursos hídricos por parte de la industria y el impacto del cambio climático. Al igual que Chile, Australia ha adoptado un sistema de mercado integrado en lo relativo a la regulación del agua; involucrando la planificación hídrica centralizada y localizada, limitando las extracciones de agua ${ }^{45}$ y permitiendo las transferencias de derechos de agua en los mercados ${ }^{46}$.

Aunque Australia es una Nación federada, históricamente los Estados han conservado la responsabilidad de gestión de los recursos hídricos, y cada uno cuenta con sus propias leyes sobre el agua ${ }^{47}$. Sin embargo, la política y regulación contemporánea del agua en Australia se ha centrado en la cuenca Murray-Darling, que abarca más de un millón de kilómetros cuadrados en el sudeste de Australia, y que suministra de agua a la mayoría de los regantes de este país, así como a muchas ciudades y una capital. Después de la década de 1980, cuando Australia sufrió severas floraciones de algas y una salinidad creciente en la cuenca, los gobiernos estatales y federal se vieron obligados a reconsiderar el enfoque australiano para la gestión ambiental del agua.

En la década de 1990, se desarrolló un enfoque nacional para la regulación del agua junto con una política de libre competencia, que buscaba facilitar la eficiencia en el uso del agua. Este enfoque de "mercado ambiental" para la regulación del agua obligó a los Estados a limitar los derechos de agua, mejorar la transparencia del precio de los títulos de aprovechamiento, separó a los derechos de agua de los títulos de propiedad inmobiliaria y estableció la libre transferibilidad de los derechos, además de asignar derechos de agua al medio ambiente ${ }^{48}$, todo con la esperanza de devolver a los ecosistemas sobreasignados, a un nivel ambientalmente sostenible. A diferencia de Chile, estos "derechos de acceso al agua", separados de la tierra, no son tratados como derechos de "propiedad", porque esto sería inconsistente con la declaración de que la Corona tiene el dominio de todas las aguas y el 'common law' no permite adquirir la propiedad del agua en su estado natural ${ }^{49}$.

A medida que empeoraba la sequía en el sudeste de Australia, en 2007 el Gobierno Federal asumió la responsabilidad de la gestión de los recursos hídricos dentro de la cuenca Murray-Darling, sobre la base de una comprensión más amplia de la gestión integrada de cuencas. Con el apoyo de los Estados, el Gobierno Federal aprobó la legislación necesaria para establecer

\footnotetext{
45 Kildea y Williams 2010; Gardner, Bartlett y Gray 2009.
}

46 Council of Australian Governments' Communique, 25 Febrero 1994, Adjunto (19 Julio 2011) <http://www.coag.gov.au/coag_meeting_outcomes/1994-02-25/docs/attachment_a.cfm>.

47 Kildea y Williams 2010; Gardner, Bartlett y Gray 2009.

48 Council of Australian Governments' Communique, 25 February 1994, Attachment A (19 July 2011) <http://www.coag.gov.au/coag_meeting_outcomes/1994-02-25/docs/ attachment_a.cfm>.

49 See, for example, Water Act 1989 (Vic), Water Management Act 2000 (NSW), Water Act 2000 (Qld), Natural Resources Management Act 2004 (SA), Rights in Water and Irrigation Act 1914 (WA), Water Act (NT) and Water Management Act 1999 (Tas). 
un límite sostenible en la extracción de agua en la cuenca y proporcionó un marco para inversiones significativas en recuperación de agua para el medio ambiente ${ }^{50}$, a través de programas de compra de derechos de agua e inversión en proyectos de eficiencia hídrica. Volúmenes sustanciales de agua ahora se reservan para fines ambientales dentro de la cuenca Murray-Darling y otros sistemas de aguas superficiales en el este de Australia, incluyendo tanto los derechos de usar agua como los límites regulatorios que protegen los caudales de agua.

\subsection{El Titular Victoriano de Agua Ambiental}

Victoria es un Estado ubicado en el sureste de Australia e incluye partes de la cuenca Murray-Darling. Su marco de asignación de agua se establece en virtud de la Ley de Agua de Victoria de 1989 y está diseñado en torno a los derechos de captar y usar agua, los que, en su mayoría, se mantienen separados de los derechos de propiedad de la tierra, y pueden comercializarse e intercambiarse en un mercado de agua ${ }^{51}$.

Victoria ha sido líder en la regulación ambiental del agua en Australia, después de haber establecido una de las regulaciones de derechos de agua ambiental más antiguas y significativas en 1999; un derecho a usar agua en el río Murray para proteger y mantener el hábitat, su flora y fauna ${ }^{52}$. Esto precedió al establecimiento formal de la "reserva de agua ambiental" de Victoria en 2005, que separa de los derechos de agua (generalmente agua en almacenamientos que pueden entregarse en sitios ambientales), caudales mínimos de agua restante "por encima del límite" para fines ambientales ${ }^{53}$. El Gobierno de Victoria también ha invertido en la recuperación de agua para el medio ambiente ${ }^{54}$, y volúmenes sustanciales de agua ambiental ahora se utilizan para lograr objetivos medio ambientales en todo Victoria.

En 2010, y en respuesta a la grave sequía continua en la cuenca y su impacto en los regantes y las especies acuáticas, el Gobierno Victoriano estableció el Titular de Agua Ambiental Victoriano (VEWH) ${ }^{55}$ para gestionar los derechos medio ambientales de agua de Victoria y así lograr mejorar los

50 Department of Sustainability, Environment, Water, Population and Communities, Water for the Future (4 July 2011) <http://www.environment.gov.au/water/australia/ index.html>.

51 National Water Commission, 2014.

52 Bulk Entitlement (River Murray - Flora and Fauna) Conversion Order 1999, in Victoria Government Gazette G24 17 June 1999, 1421.

53 Water Act 1989 (Vic), s 4A.

54 The Living Murray First Step program; Victoria's contribution from this regularisation was 120 gigalitres. State of Victoria, 'Victorian Government White Paper: Securing Our Water Future Together' (Department of Sustainability and Environment, 2004), Actions 2.3 and 3.6.

55 O' DONNell y MACPHeRson 2012. 
valores y salud de los ecosistemas acuáticos de dicho Estado ${ }^{56}$. El objetivo de esta innovación política era mejorar la independencia, la rendición de cuentas y la transparencia en la gestión ambiental del agua y, específicamente, facultar a la nueva institución para tomar decisiones sobre el uso del agua ambiental (incluido el comercio de los derechos del agua) sin interferencia política, pero aún dentro del marco de política relevante ${ }^{57}$.

Las tenencias de agua ambiental del VEWH son compatibles con un sistema basado en el mercado, ya que se componen, en gran medida, de derechos de agua que son muy similares a los derechos de agua privados. La Ley de Agua de 1989 estipula que el VEWH debe tomar una decisión cada año sobre cómo se utilizará su cartera de derechos de agua. Por ejemplo, dependiendo de los niveles de caudal dentro del sistema, puede decidir si el agua se va a dejar dentro del caudal, si se extrae para el uso de humedales o si se transmite a otros usuarios ${ }^{58}$.

El VEWH es una corporación legal y, por lo tanto, una "persona jurídica". Su legislación habilitante establece que el VEWH es un "cuerpo corporativo", que puede "demandar o ser demandado en su nombre corporativo (...) y puede realizar y soportar todos los actos y contratos que un cuerpo corporativo puede hacer y soportar por ley"59. Esto le otorga al VEWH una posición legal de derecho propio en caso de cualquier disputa y la facultad de celebrar contratos y tratar con bienes muebles o inmuebles ${ }^{60}$.

Tal y como sucede en el caso de las Juntas de Vigilancia en Chile, aunque el VEWH ostenta el beneficio de la personalidad jurídica, los ríos que administra no lo hacen. Sin embargo, a diferencia de Chile, los valores de los ríos gozan de protección legal expresa ya que dentro de los objetivos del VEWH se establece que este debe propender a "mejorar los valores ambientales y la salud de los ecosistemas acuáticos, incluyendo su biodiversidad, funcionamiento ecológico y calidad del agua, y otros usos que dependen de la condición ambiental" "61. Además, los derechos medio ambientales del agua que posee el VEWH establecen expresamente que el agua asignada en virtud de estos derechos se utilizará para lograr resultados medio ambientales ${ }^{62}$. Esto minimiza el potencial de divergencias entre los intereses del VEWH y los intereses ambientales de los ríos.

De hecho, el VEWH está "aislado", en gran medida, de la interferencia del Ministro de Medio Ambiente de Victoria sobre cómo el primero decide

56 Water Act 1989 (Vic), ss33DC-DE.

57 O'DONNELL 2012.

58 O’DONNELL 2013.

59 Water Act 1989 (Vic), s33DB.

60 O'Donnell 2013; O'Donnell y Garrick 2017.

61 Water Act 1989 (Vic), s33DC (b).

62 See, for example, Bulk Entitlement (Thomson River - Environment) Order 2005; Yarra Environmental Entitlement 2006. 
usar su agua, siempre que tal uso esté de acuerdo con sus objetivos ambientales expresos. Si bien el VEWH es responsable ante el Ministro de Medio Ambiente de Victoria, y debe informar a este y cumplir con las normas ministeriales, la autoridad gubernamental no puede emitir ninguna instrucción sobre cómo se gestionarán las tenencias de agua ambiental en un año determinado63. Por ejemplo, el Ministro no puede ordenarle al VEWH que use agua en un lugar en particular o para un propósito determinado (o que no lo haga), o si debe comprar o vender derechos de agua. O'Donnell ha señalado que el VEWH actúa como un "guardián" de los caudales ambientales internos, trabajando con otros titulares de aguas ambientales y autoridades de gestión de cuencas para determinar dónde, cuándo y cómo usar el agua para el medio ambiente en el Estado de Victoria ${ }^{64}$.

\section{Te Awa Tupua}

\subsection{La regulación del agua en Nueva Zelanda}

En contraste con Australia, Nueva Zelanda tiene una precipitación anual alta $y$, aunque las condiciones varían considerablemente entre las diferentes zonas del país, en promedio, la cantidad de agua no es una preocupación importante ${ }^{65}$. Sin embargo, la reputación de Nueva Zelanda como "limpia y verde" no es del todo exacta, y en muchos lugares los ríos de Nueva Zelanda sufren por tener un caudal irregular y una mala calidad de agua como resultado de los grandes impactos agrícolas e industriales ${ }^{66}$.

Es un mérito el que Nueva Zelanda goce de leyes y políticas de gestión de recursos naturales altamente integradas en virtud de la Ley de Gestión de Recursos (en adelante LGR) de 199167. Además de proporcionar una "ventanilla única" para autorizaciones y permisos en orden a utilizar recursos naturales, la LGR adopta un enfoque ecosistémico respaldado por el concepto de "gestión sostenible"68. A diferencia de Australia y Chile, el enfoque de Nueva Zelanda sigue siendo un modelo de asignación de agua "planificado", en lugar de un mercado integrado, con una baja incidencia del comercio de agua ${ }^{69}$.

Al igual que Australia, y en virtud del "common law" neozelandés, nadie puede "ser propietario" del agua, ya que esta es de propiedad de la Corona quien la ostenta en nombre del pueblo de Nueva Zelanda. Sin embargo, en virtud de la LGR, los concejos municipales toman la decisión de otorgar una "autorización de recursos" para extraer y usar las aguas bajo el paradigma de

63 Ibid ss33DS, 33DT, 33DZA.

64 O'DONNELL 2017; O'DONNELL 2013.

65 New Zealand Ministry for the Environment, 2016: 5-6.

66 OECD, 2017.

67 Warnock y BaKer-Galloway 2014, 17.

68 Resource Management Act 1991 (NZ) s 5.

69 OECD, 2017; New Zealand Ministry for the Environment, 2016: 40-1. 
"quien llega primero, se sirve primero". Estas autorizaciones son temporales, aplicando solo hasta los 35 años pero, por lo demás, tienen muchas de las mismas características que los derechos de aprovechamiento de Chile o de los "derechos de acceso al agua" en Australia.

La LGR incluye una gama de mecanismos para proteger los valores ambientales y culturales del agua. La ley exige expresamente que los concejos municipales de autorización reconozcan y garanticen la "relación de los maoríes, su cultura y tradiciones con sus tierras ancestrales, agua, sitios, waahi tapu [lugares sagrados] y otros taonga [tesoros]" (S 6), y el concepto de "kaitiakitanga" [tutela] (S 7). La Ley también permite a los concejos municipales concertar acuerdos de gobernanza en colaboración con los maoríes y sus iwi y hapū (tribus y subtribus) sobre los recursos naturales o para delegar la toma de decisiones en la gestión de los recursos a los grupos maoríes ${ }^{70}$. Esos acuerdos de "cogestión" se han utilizado en el pasado para los ríos, a menudo como consecuencia de las reclamaciones maoríes de reparación en virtud del Tratado de Waitangi, incluido el río Waikato ${ }^{71}$. El Segmento 9 de la ley también permite a cualquier persona solicitar al Ministro de Medio Ambiente una "orden de conservación del agua" para proteger los valores ambientales o culturales del agua. Sin embargo, a pesar de estas protecciones, en muchas partes de Nueva Zelanda, la concesión y el uso de los recursos naturales han conducido a la contaminación, eutrofización y extracción excesiva de agua desde los ríos, o han tenido un impacto en la relación espiritual o física de los maoríes con sus aguas.

\subsection{Te Awa Tapua}

En marzo de 2017, Nueva Zelanda se convirtió en el primer país en aprobar una legislación que reconoce a un río como una persona jurídica. Esto, como parte de un acuerdo político con Whanganui Iwi ${ }^{72}$ que, tradicionalmente, ha utilizado y mantenido relaciones con el río en cuestión. En lugar de tratar al río como un recurso para ser explotado por la gente de Whanganui, en la Ley de Te Awa Tupua, los Whanganui Iwi se posicionan como interdependientes y corresponsables del río. Sin embargo, a pesar de sus materias ecocéntricas, el modelo es antropocéntrico en el sentido de que los derechos del río bajo la Ley Te Awa Tupua están intrínsecamente vinculados con los derechos al río de los Whanganui Iwi y su "diferencia" cultural indígena".

70 Resource Management Act 1991 (NZ) ss 58L-58U (called Mana Whakahono a Rohe).

71 Waikato-Tainui Raupatu Claims (Waikato River) Settlement Act 2010 (NZ).

72 En el presente paper, por simplicidad, nos referimos a los iwi como 'Whanganui River Iwi', aunque sabemos que el número de otras iwi (tribus) y hapū (subtribus) tienen intereses en el río Whanganui.

73 Ver Magallanes 2015, 273. 
La Ley Te Awa Tupua también es un arreglo político reparador ${ }^{74}$ de una de las disputas más antiguas de Nueva Zelanda sobre la gestión y propiedad de los ríos, como consecuencia de la privación histórica, la degradación ambiental y el desarrollo no equitativo ${ }^{75}$. En el contexto de Nueva Zelanda, los acuerdos políticos sobre los ríos (y el agua en general) no son nuevos ${ }^{76}$. El Tribunal de Waitangi ha conocido, y la Corona ha negociado y resuelto, reclamaciones sobre ríos y lagos en numerosas ocasiones durante los últimos 40 años, incluido el río Waikato, que fue reconocido como un "antepasado vivo" y un "todo indivisible" en una manera similar a Te Awa Tupua, aunque sin personalidad jurídica ${ }^{77}$. Los acuerdos fluviales anteriores se han centrado en otorgar a los maoríes derechos de "cogestión" o un derecho a participar activamente en la gobernanza y regulación de los ríos, junto con la Corona, de conformidad con el principio de asociación del Tratado (una relación típicamente descrita por los maoríes bajo el concepto de "kaitiakitanga" o tutela).

Sin embargo, la Ley Te Awa Tupa va más allá de los acuerdos fluviales anteriores y específicamente declara que el río es una "persona jurídica". Tras el reconocimiento del bosque de Te Urewera como "persona jurídica", en un arreglo político celebrado a propósito de una reclamación interpuesta ante el Tribunal de Waitangi por otro grupo de maoríes en $2014^{78}$, y el reciente acuerdo que otorga personalidad jurídica a la Montaña Taranaki en diciembre 2017 la Ley Te Awa Tupua es indicativa de una tendencia hacia el uso de la personalidad jurídica en la reparación cultural y, en última instancia, establece marcos de gobernanza para los recursos naturales en Nueva Zelanda.

La Ley Te Awa Tupua reconoce el estado del río Whanganui (y sus afluentes) como "un todo indivisible y vivo, que comprende el río Whanganui desde las montañas hasta el mar, incorporando todos sus elementos físicos y metafísicos"79. Este encuadre de Te Awa Tupua refleja el tikanga maorí (ley consuetudinaria maorí) del Whanganui $I w i^{80}$, y es consistente con el enfoque "territorial" de los recursos naturales en el derecho internacional indígena. Se puede contrastar con las concepciones liberales occidentales de los recursos

74 Macpherson 2016.

75 Las tribus Whanganui mantuvieron su derecho de propiedad sobre el río a través de una serie de casos judiciales desde 1938 a 1962 y a través de una consulta al Tribunal Waitangi que conllevó al Informe del Río Whanganui en 1999 (Trbunal Waitangi 1999). Las preocupaciones de los Maorí sobre la pérdida de sus derechos de propiedad, administración y control sobre el río fueron exacerbadas por la degradación medioambiental infligido en él a propósito de las faenes mineras, el desarrollo hidroeléctrico y una pobre administración en los años que siguieron a la colonización.

76 Ruru 2013 340, Magallanes 2015, 309.

77 Waikato-Tainui Raupatu Claims (Waikato River) Settlement Act 2010 (NZ).

78 Te Urewera Act 2014 (NZ).

79 Te Awa Tupua Act (NZ) s 12.

80 Hutchison 2014, 180. 
naturales como objetos divisibles para la propiedad y la regulación. Esto es evidente a partir de la inclusión de los elementos "metafísicos" del río en su estado, junto con el físico, aludiendo a las conexiones espirituales y culturales entre el Whanganui Iwi y el río. Sin embargo, la conceptualización del río como un "todo indivisible y vivo" también se alinea con un enfoque de "cuenca integrada" para la regulación de los ríos.

La Ley Te Awa Tupua luego "declara" que Te Awa Tupua es una "persona jurídica", que tiene "todos los derechos, facultades, deberes y responsabilidades de una persona jurídica" 81 . El uso de "declara" aquí es interesante, ya que se evita el concepto "reconoce", usado en la Sección 12 (cuando se señala que el río es "Te Awa Tupua"). De esta manera, la Ley explicita el estado preexistente del río como Te Awa Tupua, pero evita decir expresamente que el río ya es (intrínseca o justificablemente) una persona jurídica. Al usar la palabra "declara", en lugar de la palabra "otorga", la Ley también evita el extremo de parecer que confiere personalidad jurídica al río, dejando abierta la posibilidad de que alguien pueda argumentar que un río puede ser una persona en tikanga maorí, independientemente de si el Estado lo reconoce como tal.

Como se mencionó anteriormente, los "derechos, deberes y obligaciones" de una persona jurídica son los derechos a demandar, ser demandado, celebrar actos y contratos, poseer bienes y ser titular de propiedades. Por supuesto, el río en sí mismo no puede litigar ante tribunales o comprar tierras, por lo que la Ley Te Awa Tupua crea un representante, llamado "Te Pou Tupua", que debe actuar en interés del río. La "oficina" de Te Pou Tupua, establecida por la ley, es la "cara humana" del río para actuar en nombre y representación de Te Awa Tupua, como una especie de guardián del río; uno de sus guardianes es nominado por la Corona y otro por el río Whanganui Iwi ${ }^{82}$. El modelo de centinela adopta un enfoque ecocéntrico para la gestión de los recursos naturales, habiendo propuesto Stone un modelo de tutela para proteger los derechos de la naturaleza ${ }^{83}$. Así las cosas, el modelo de guardián también refleja el "principio de asociación" ("partnership") con los maoríes en virtud del Tratado de Waitangi ${ }^{84}$. Sin embargo, el modelo de tutela adoptado en Te Pou Tupua ciertamente va más allá de las aplicaciones anteriores del "principio de asociación" en cuanto a acuerdos de gestión conjunta de recursos naturales, en los que el Gobierno Central o las autoridades locales/ regionales comparten la administración pública de recursos naturales con grupos maoríes. Te Pou Tupua le da a los maoríes un papel más importante

\footnotetext{
81 Te Awa Tupua Act (NZ) s 14.

82 Te Awa Tupua Act (NZ) ss 18-19.

83 StONe 2010.

84 Magallanes 2015, 281.
} 
en la gobernanza del agua, en reconocimiento de los derechos de autogobierno maorí85, en la forma de un asiento igualitario en la mesa de gobierno.

Te Pou Tupua tiene amplias facultades, aunque debe actuar en interés del río y de forma coherente con los valores prescritos para la gestión del mismo. Estos principios (llamados "Tupua te Kawa") se establecen en la Sección 13 de la Ley, que los describe como los "valores intrínsecos que representan la esencia de Te Awa Tupua". Estos valores son muy importantes ya que nos ayudan a comprender el contenido de los derechos del río ${ }^{86}$. Los encargados de la toma de decisiones administrativas se encuentran supeditados a la legislación que regula la gestión del río y (incluyendo, en particular, la LGR de 1991) deben reconocer, prever y tener en cuenta el estado de Te Awa Tupua como persona jurídica y los valores estatutarios del río, como consideración relevante ${ }^{87}$. Sin embargo, la Ley Te Awa Tupua señala que las decisiones tomadas en virtud de otra legislación deben ser ejercidas de acuerdo con el propósito de esa legislación y ni el estado de Te Awa Tupua ni Tupua te Kawa pueden ser factores determinantes en la decisión administrativa ${ }^{88}$, generando una percepción de "sello de goma".

Los valores estatutarios del río, "Tupua te Kawa", reconocen al río como una fuente de sustento espiritual y físico, manteniendo los recursos dentro de él y a las personas que viven en sus riberas ${ }^{89}$. Estos principios reconocen el vínculo directo entre la salud del río y de las personas, lo que refleja la evidencia presentada ante el Tribunal Waitangi que conduce a la solución de que la contaminación de la espiritualidad del río afecta negativamente a la salud de las personas ${ }^{90}$. Los principios reiteran que el río es un todo indivisible y vivo desde las montañas hasta el mar, incorporando elementos físicos y metafísicos. Los principios también reconocen la responsabilidad del Whanganui Iwi por la salud del río (tutela), que los arroyos grandes y pequeños forman un solo río (gestión integrada de cuencas) y que el propósito común de todos los elementos y entidades del río es trabajar para la salud y bienestar del mismo.

Finalmente, la Ley Te Awa Tupua establece un complejo régimen de gestión colaborativo entre los maoríes, el Gobierno Municipal y Central, y los usuarios privados ${ }^{91}$. En este régimen, participan una amplia gama de entida-

\footnotetext{
85 Such rights are reflected in the guarantee of tino rangatiratanga under article 2 of the Treaty of Waitangi.

${ }_{86}$ Good 2013, 35-6. Good discute la dificultad, una vez que los derechos de la naturaleza son reconocidos, de determinar el contenido de tales derechos, en ausencia de la capacidad del río de hablar por sí mismo.

87 Te Awa Tupua (Whanganui River Claims Settlement Act) 2017 (NZ) s 15.

88 Te Awa Tupua (Whanganui River Claims Settlement Act) 2017 (NZ) s 15(5).

89 Te Awa Tupua Act (NZ) s 13(a).

90 Tribunal Waitangi 1999.

91 Julia TALBOt-JONES 2016.
} 
des y se abarcan una variedad de perspectivas, no solo las de Te Pou Tupua o Whanganui River Iwi. En particular, no se requiere de la autorización de Te Pou Tupua para presentar las solicitudes de permisos de recursos en orden a utilizar el agua del río según la Ley de Gestión de Recursos de 1991 (se requiere de su consentimiento para usar el lecho del río), aunque un concejo municipal pueda determinar que Te Pou Tupua es una "persona afectada"92. De hecho, aún no se ha visto cómo el modelo de Te Awa Tupua funcionará en la práctica, ya que el marco político circundante no se ha desarrollado completamente.

Lo que no hace la Ley Te Awa Tupua es impactar, de alguna manera, los intereses de propiedad en el río. El acuerdo es la culminación de más de un siglo de agitación indígena por el derecho a la "propiedad" del río, siempre resistido por la Corona sobre la base de que "nadie puede ser dueño del agua". Según la Ley, solo las partes del lecho del río que son propiedad de la Corona están conferidas a Te Awa Tupua, y el acuerdo no tiene ningún impacto en los derechos públicos de uso, pesca o navegación, o las autorizaciones o permisos privados para usar el río, según una disposición que protege específicamente los derechos de los generadores de energía hidroeléctrica como de empresas estatales y compañías de modelos de uso mixto ${ }^{93}$. De esta manera, la Ley Te Awa Tupua sigue el enfoque adoptado en una serie de acuerdos anteriores, que otorgaron los lechos de ríos y lagos a grupos maoríes y se centraron en la cogestión.

\section{La personalidad jurídica ¿brinda la oportunidad de mejorar los re- sultados ambientales en la regulación de los ríos?}

Si la Ley Te Awa Tupua crea una institución para actuar en interés del río, de acuerdo con principios legales específicos, ¿qué oportunidades ofrece el otorgamiento de personalidad jurídica, más allá de lo que otras organizaciones relacionadas con los ríos (para ríos sin personalidad jurídica propia) son capaces de proporcionar? En ausencia de un marco político, y previo someterse a prueba ante tribunales, no podemos saber con precisión qué beneficios adicionales podrían obtenerse. Sin embargo, en esta sección se exploran algunas oportunidades posibles, aprovechando este estudio de modelos de Australia, Chile y Nueva Zelanda. Como se mostrará, los tres modelos caen en un espectro, desde aquel con menor potencial de resultados ambientales (Chile), hasta el que presenta mayor potencial (Nueva Zelanda). El modelo australiano se encuentra en el medio, como un intento significativo de dar voz al medio ambiente en la asignación y gestión de los ríos en Victoria, pero sin la concesión directa de personalidad jurídica a los ríos. La siguiente tabla muestra cómo los tres modelos difieren en aspectos importantes, los que tie-

\footnotetext{
92 Resource Management Act 1991 (NZ) s 46(3).
}

93 Resource Management Act 1991 (NZ) ss 41, 6. 
nen diferentes grados en la efectividad de cada uno de ellos en términos de oportunidades para los resultados ambientales en la regulación de los ríos.

Tabla No 1 Derechos y regulación de los ríos en un espectro en Chile, Australia y Nueva Zelanda.

\begin{tabular}{|c|c|c|c|}
\hline $\begin{array}{c}\text { Atribuciones } \\
\text { legales e institu- } \\
\text { cionales }\end{array}$ & $\begin{array}{c}\text { Juntas de } \\
\text { Vigilancia } \\
\text { (Chile) }\end{array}$ & $\begin{array}{c}\text { Titular Victoriano } \\
\text { de Agua Ambiental } \\
\text { (Australia) }\end{array}$ & $\begin{array}{c}\text { Te Awa Tupua } \\
\text { (Nueva Zelanda) }\end{array}$ \\
\hline $\begin{array}{c}\text { La entidad es una } \\
\text { persona jurídica. }\end{array}$ & Sí & Sí & Sí \\
\hline $\begin{array}{c}\text { El río es una per- } \\
\text { sona jurídica. }\end{array}$ & No & No í \\
\hline $\begin{array}{c}\text { Los valores del } \\
\text { río están expresa- } \\
\text { mente protegidos } \\
\text { en la ley. }\end{array}$ & No & Sí & Sí \\
\hline $\begin{array}{c}\text { La entidad per- } \\
\text { mite una gestión } \\
\text { integrada de } \\
\text { cuenca. }\end{array}$ & Sí & No & Sí \\
\hline $\begin{array}{c}\text { La entidad/río es } \\
\text { titular de derechos } \\
\text { de aprovecha- } \\
\text { miento de aguas }\end{array}$ & Sí & Sí & No \\
\hline
\end{tabular}

Una posible fortaleza ambiental de las Juntas de Vigilancia chilenas, el más débil de los tres modelos en términos de oportunidades para los resultados ambientales, es el hecho de que pueden abarcar una gestión de cuenca hidrográfica completa e integrada. Las Juntas, como personas jurídicas, sí tienen voz y la capacidad de emprender acciones legales a nombre propio. Sin embargo, es probable que lo hagan cuando esto coincida con los intereses de los accionistas que manejan la titularidad de sus derechos de agua; usuarios que generalmente son irrigadores. La función pública de "resolución de conflictos" que estas organizaciones desempeñan con preferencia a los tribunales de justicia se refiere a las disputas entre los usuarios de agua en materias de distribución y uso del recurso ${ }^{94}$. Como ejemplo de las demandas formuladas por las Juntas, existe una decisión reciente del Tribunal Ambiental de Santiago entablada (sin éxito) por la Junta de Vigilancia del Río Mapocho, por supuesto daño ambiental en el que se redujeron caudales por un

94 Ríos Brehm y QUiROz 1995, 3. 
gran proveedor de agua residencial, centrándose en el impacto de caudales reducidos a pequeños agricultores ${ }^{95}$.

La relativa debilidad del modelo de Juntas de Vigilancia se ve agravada por el hecho de que estas no poseen los derechos de aprovechamiento, que son propiedad de sus accionistas individuales. Las Juntas no pueden incorporar los intereses del río, ni los intereses de aquellos sin derechos de aprovechamiento formales, tales como comunidades indígenas o consuetudinarias, grupos de interés público y organizaciones no gubernamentales, ni resultados ambientales del río o incluso el interés público ${ }^{96}$. Debido a que el Código de Aguas asume que el agua se usará para usos efectivos, los usos ambientales o culturales no están típicamente representados por derechos de aprovechamiento formales, y por lo tanto, los intereses ambientales no están representados en las participaciones. El potencial que tiene un gran accionista, un generador de energía hidroeléctrica o una mina, por ejemplo, para dominar la toma de decisiones en una Junta de Vigilancia es significativo. En esta situación, convertir al río en una persona jurídica le daría una voz distinta para visibilizar sus intereses ambientales.

El VEWH, también una persona jurídica, podría tomar medidas coherentes con los intereses de un río, dado que se rige por objetivos medioambientales expresos y opera a distancia del Gobierno. Sin embargo, a diferencia del modelo chileno, el VEWH no proporciona una gestión integrada en la cuenca o en la escala de captación, y aunque posee una cartera sustancial de derechos de acceso al agua, solo es titular en una cuenca, sujeto a la presión de múltiples usuarios y regímenes reguladores. Lo más importante es que el VEWH solo puede actuar en nombre de los ríos en los que es titular de derechos de agua, lo que cubre solo una pequeña porción del agua total asignada al medio ambiente (bajo la reserva de agua ambiental). Si los ríos de Victoria tuvieran personalidad jurídica propia, todos ellos podrían actuar por sí mismos y participar en debates sobre políticas públicas de gestión ${ }^{97}$. Sin embargo, como titular de derechos de agua, el VEWH tiene una facultad real para influir en la regulación del agua en los ríos victorianos, como comprador y vendedor de derechos, y al decidir dónde y cuándo usar esa agua en beneficio del medio ambiente. Por lo tanto, una de las principales fortalezas del VEWH, como entidad de gestión fluvial, proviene del hecho de que posee derechos constituidos legalmente sobre las aguas, y se establece, legalmente, que use esos derechos para mejorar la salud del ambiente acuático.

Esto nos lleva a un hallazgo clave: que los derechos legalmente consagrados a los ríos pueden ofrecer una oportunidad para mejorar la regulación ambiental cuando existe una brecha en la protección de estos, porque las

95 Junta de Vigilancia de la Tercera Sección del Río Mapocho con Aguas Andinas S.A:

27 Abril 2017, Segundo Tribunal Ambiental (D-24-2016) (Chile).

96 Prieto y Bauer 2012, 141-2.

97 O'DONNELL 2012. 
leyes e instituciones existentes no resguardan los intereses del río. Esto se debe, generalmente, a que los intereses del río no se alinean con los intereses de los reguladores o usuarios existentes, o cuando los intereses están demasiado fragmentados para proporcionar un enfoque holístico. En otras palabras, la personalidad jurídica agrega valor cuando un río en particular, en sus circunstancias características, necesita una voz y capacidad para ser escuchado, para competir en los resultados. Por esta razón, la personalidad jurídica se ha adoptado en contextos en los que los ríos están sujetos a una gran amenaza de degradación y los gobiernos no han podido responder eficazmente utilizando los mecanismos existentes.

A diferencia de los otros dos modelos, el de Te Awa Tupua, de Nueva Zelanda, otorga personalidad jurídica al río. Esto permitiría que el río emprenda acciones legales a nombre propio, en caso de ser necesario, para responder a las amenazas o para promover los resultados ambientales. La idea de extender la personalidad jurídica a un río se alinea con un enfoque ecocéntrico en la regulación de los recursos naturales, sin embargo, en el contexto de Nueva Zelanda, también se deriva del pluralismo cultural y legal; reflejando la conceptualización de la relación entre los humanos y el mundo natural en el tikanga (ley consuetudinaria maorí) de Whanganui Iwi. Según la cosmovisión de Whananui Iwi, el río tiene los mismos derechos que pertenecen a los Iwi y no al revés, ejemplificado en el idioma Iwi: "Yo soy el río y el río soy yo". Por supuesto, la Ley Te Awa Tupua es antropocéntrica en el sentido de que la personalidad jurídica es parte de un acuerdo político con Whanganui Iwi, que tiene la intención de reparar los errores del pasado y reconocer las relaciones culturales maoríes con el agua ${ }^{98}$.

Sin embargo, la falla en darle al río el derecho a su propia agua crea una paradoja. ¿Cómo puede Te Awa Tupua ser un "todo indivisible y viviente desde las montañas hasta el mar incorporando elementos físicos y metafísicos" y aún dividirse en diferentes regímenes propietarios para el uso del suelo, el agua, la vida silvestre, etc.? ¿Cómo puede la Ley Te Awa Tupua ser presentada por algunos defensores de los derechos consagrados legalmente a los ríos como ilustrativa de la dicotomía entre propiedad de la naturaleza y derechos para la naturaleza, cuando el modelo no tiene impacto sobre los regímenes de derechos legales subyacentes (y superpuestos) ${ }^{99}$ ?

Podríamos ver a Te Awa Tupua afirmando sus intereses como persona jurídica en el futuro, por ejemplo, al solicitar una orden de conservación del agua para proteger el río. Pero sin que el río tenga derecho a usar su agua, y en el contexto de poderosos derechos de uso público y privado sobre el agua en él, es fácil ver cómo los intereses del río pueden subordinarse a los de sus usuarios. ¿Qué sucede cuando un generador de energía hidroeléctrica ejercita sus derechos para utilizar el agua del río Whanganui de una manera

\footnotetext{
98 Magallanes 2015, 325-6.

99 HUtCHISON 2014, MARGIL 2017.
} 
que impacta negativamente la salud física o espiritual del río? ¿Puede Te Pou Tupua tomar acción para la protección ambiental del río? La Ley Te Awa Tupua es un estatuto de rango ordinario (no constitucional), con la incapacidad de anular los derechos otorgados en virtud de otra legislación. Todavía no hemos visto ningún litigio sobre la reciente innovación jurídica, sin embargo, dado el potencial de conflicto sobre el uso de aguas del río, el éxito del modelo de Te Awa Tupua dependerá no solo de una fuerte gobernanza colaborativa, sino de la capacidad de los tribunales para resolver disputas. Con suerte, las actuales reclamaciones de los maoríes a nivel nacional sobre la propiedad del agua están frente al Tribunal de Waitangi y se resolverán en el futuro. Mientras tanto, está claro que las dicotomías tradicionales que diferencian los derechos de la naturaleza de los derechos a la propiedad, son excesivamente simplistas y potencialmente engañosas.

Las reclamaciones hechas por los maoríes a propósito del río Whanganui, siempre se habían enmarcado como derechos de "propiedad", y el Tribunal de Waitangi que conoció sobre esas reclamaciones no mencionó, ni una vez, los conceptos de "derechos para la naturaleza" o "personalidad jurídica". Enmarcó los intereses de los maoríes en el río como de naturaleza "propietaria" y "territorial", aunque reconoció la relación de los maoríes con el río como una especie de ascendencia ${ }^{100}$. Tomado en contexto, la Ley Te Awa Tupua es un compromiso político, que no le permite a nadie el derecho de "dominio" del río como un todo"101. Evitando el complicado tema de la redistribución de los derechos de uso, ya sea hacia el Whanganui Iwi o el río mismo, el Modelo Te Awa Tupua es realmente una propuesta avanzada de regulación colaborativa, en el que se enfatizan los intereses del río, de los maoríes y otros intereses comunitarios y comerciales.

Sin embargo, aparte de las posibles vías legales de reparación ofrecidas por la situación legal, el reconocimiento de un río como una "persona" permite un cambio cultural crucial en la forma en que pensamos los ríos. El enfoque de persona jurídica trasciende la conceptualización occidental del río como una conjunción de partes en movimiento. El río se ve como una entidad interconectada, y los aspectos regulatorios sobre ciertos sectores del río deberían considerar el impacto en otros. Quizás lo más importante es que el río se respeta como un fin en sí mismo, por principios similares a los que mantienen los pueblos indígenas. Ello es evidente en el Tupua te Kawa, o valores del río, adoptado para el río Wanganui. Esto, solo, podría cambiar de manera subversiva nuestro enfoque sobre la gestión de ríos, en el desarrollo de políticas públicas y ante los tribunales.

Esta discusión nos lleva a algunas condiciones clave para que la personalidad jurídica mejore la regulación ambiental, según corresponda. La primera de ellas es la importancia de que existan instituciones fuertes para

100 Tribunal Waitangi, 1999: 337, 343.

101 HARDCASTLE, 2014. 
hacer cumplir y defender los derechos del río; que estén protegidas, lo más posible, de la interferencia política o privada. Con el fin de minimizar la influencia de los usuarios que compiten, la segunda condición es clara, y consiste en expresar valores para el río, preferiblemente consagrados por la legislación. Debido a que el río actúa a través de un representante, es importante el poder responsabilizar a este para que actúe en función de los intereses del río. Si terceros pudieran solicitar a los tribunales que respeten los derechos del río, esto mejoraría aún más la protección y alentaría la protección activa del río. La tercera y última condición para mejorar los resultados ambientales a través del uso de la personalidad jurídica son los derechos legalmente constituidos en el río. Si otros tienen intereses legales o de propiedad en los ríos y este no, el alcance para mejorar los valores ambientales en la regulación de los ríos se ve seriamente socavado.

\section{Conclusión}

La regulación de los ríos siempre se ha basado en la premisa de que estos recursos naturales son valiosos debido al beneficio que brindan a los seres humanos, en el apoyo al desarrollo económico y la salud humana. Esta visión asegura la posición de los seres humanos como independientes y superiores a la naturaleza, en el "centro de la preocupación" de la ley hecha por el hombre ${ }^{102}$. Sin embargo, hay un cambio de énfasis en algunas nuevas leyes de recursos naturales, como la Ley Te Awa Tupua, que prioriza la ecología por encima de la utilidad ${ }^{103}$. La declaración de que un río sea una persona jurídica no puede ser meramente simbólica. Hemos argumentado aquí que la personalidad jurídica ofrece una oportunidad para mejorar la protección de los ambientes ribereños, siempre que la personalidad jurídica del río esté respaldada por instituciones sólidas, principios legales, derechos legalmente consagrados ( $y$, en última instancia, seres humanos comprometidos) para permitir la aplicación efectiva de los intereses del río. Esto es significativo, porque en un mundo cada vez más competitivo y degradado, la personalidad jurídica ofrece una nueva vía para agitar y presionar los ánimos a favor de los intereses del medio ambiente. Donde las leyes y las instituciones existentes nos han fallado, debemos darle a la naturaleza la oportunidad de optar por la personalidad jurídica.

\section{Bibliografía citada}

[En línea] Incertidumbre por reforma al Código de Aguas. Disponible en http://www.latercera.com/noticia/incertidumbre-reforma-al-codigo-aguas/ (agosto de 2017).

BAuer, C. (2004) Siren Song: Chilean Water Law as a Model For International Reform. Resources for the Future.

BerRY, T. (1999) the Great Work: Our Way into the Future / Thomas Berry. S.L., New York: Bell Tower, C1999.

102 Morgera y Kulovesi 2016, 23. Berry 1999, 4.

103 De LuCia 2013, 170. 
Biblioteca del Congreso Nacional de Chile (2017): "Estado Actual de Proyecto de Ley Que Introduce Modificaciones al Marco Normativo que Rige las Aguas en Materia de Fiscalización y Sanciones (Boletín N 8.149-09)". Fecha de consulta: marzo 2016.

Biblioteca Nacional del Congreso (2016): "Proyecto De Reforma Constitucional sobre Dominio y Uso de las Aguas". fecha de consulta: octubre 2016.

Consejo Nacional de Innovación para el Desarrollo (2016): "Ciencia E Innovación para los Desafíos del Agua en Chile: Agenda Estratégica de Investigación, Desarrollo e Innovación para la Sostenibilidad de los Recursos Hídricos".

Davies, M; Naffine, N. (2001): Are Persons Property? Legal Debates about Property and Personality.

AldeRshot: Ashgate Publishing.

DE LuCIA, V. (2013): "Towards an Ecological Philosophy of Law: A Comparative Discussion". Journal of Human Rights \& the Environment, año 4, № 2, septiembre, 167 pp.

Donnelly, B. y BISHOP, P. (2007): "Natural Law and Ecocentrism". Journal of Environmental Law. Año 19, № 1, abril, pp. 89-101.

ECLAC y OECD (2016): oecd Environmental Performance Reviews: Chile 2016. S.L., OECD Publishing.

ElDER, P. S. (1984): "Legal Rights for Nature-The Wrong Answer to the Right (S) Question". Osgoode Hall Lj. Año 22, 285 pp.

Good, M. (2013): "The River as a Legal Person: Evaluating Nature Rights-Based Approaches to Environmental Protection in Australia". National Environmental Law Review. S.D. $N^{\circ} 1,34$ pp.

Gulloff, M (2012): "A Pragmatic Approach to Multiple Water Use Coordination in Chile". 37(2) Water International 121.

HardCAstle, L. (2014): "Turbulent Times: Speculations about the Whanganui River's Position as a Legal Entity". Maori Law Review. N February.

Hutchison, A. (2014): "Whanganui River as a Legal Person, the [Article]". Alternative Law Journal (Gaunt). S.D. N 3, 179 pp.

ImRan, S.; Alam K. y Beaumont N. (2014): "Reinterpreting the Definition of Sustainable Development for a More Ecocentric Reorientation: Reinterpreting the Definition of Sustainable Development". Sustainable Development. Año 22, № 2, marzo, pp. 134-144.

MACPHERSON (2017): Beyond Recognition: Lessons from Chile for Allocating Indigenous Water Rights in Australia 40(3) Unswlj.

Magallanes, C. J. I. (2015): "Maori Cultural Rights in Aotearoa New Zealand: Protecting the Cosmology That Protects The Environment." Widener Law Review. Año 21, № 2 , Septiembre, pp. 273-327.

Maloney, M. (2016) "Building an Alternative Jurisprudence for the Earth: The International Rights of Nature Tribunal". Vermont Law Review. S.D. No 1, 129 pp.

Maloney, M. y Burdon P. (2014): Wild Law - In Practice. [Electronic Resource]. S.L., Hoboken: Taylor and Francis.

MARGIL, M. (2017): "The Standing Of Trees: Why Nature Needs Legal Rights.". World Policy Journal. Año 34, No2, pp. 8-11.

Morgan (2007): The Intersection of Rights and Regulation: New Directions in Sociolegal Scholarship. Morgan, B. (Ed.), Aldershot, Ashgate.

Morgan, B. (2007): The Intersection of Rights and Regulation: New Directions in Sociolegal Scholarship. Aldershot, Ashgate.

Morgera, E. y KULOVESI K. (2016): Research Handbook on International Law and Natural Resources. [Electronic Resource]. S.L., Northampton, Ma: Edward Elgar Pub., C. 2016.

New Zealand Y Ministry for the Environment (2016): Next Steps for Fresh Water: Consultation Document.

O'Donnell, E. y Talbot-Jones, J. (2017): Three Rivers Are Now Legally People - But That's Just the Start of Looking after Them. The Conversation [En Linea]. 
O'Donnell, E. y Macpherson E. (2012): "Challenges and Opportunities for Environmental Water Management in Chile: An Australian Perspective". En Journal of Water Law. Año 23, No 1, pp. 24-36.

O'DonnelL, E. (2017): 'Constructing the Aquatic Environment as a Legal Subject: Legal Rights, Market Participation, and the Power of Narrative'. University of Melbourne.

OECD (2017): Oecd Environmental Performance Reviews: New Zealand 2017. S.L., OECD Publishing.

Office of Treaty Settlements (2004): Healing the Past, Building A Future: / A Guide to Treaty of Waitangi Claims and Negotiations with the Crown $=$ Ka Tika $\bar{A}$ Muri, Ka Tika $\bar{A}$ Mua: He Tohutohu Whakamārama I Ngā Whakataunga Kerēme E Pā Ana Ki Te Tiriti O Waitangi Me Ngā Whakaritenga Ki Te Karauna.

Parsons Álvarez, Franklin (2002): 'La Aguas en la Ley 19.253, de 1993, Ley Indígena' 4(1) Revista de Derecho Administrativo Económico, 117 pp.

Prieto, M. y Bauer C. (2012): "Hydroelectric Power Generation in Chile: An Institutional Critique of the Neutrality of Market Mechanisms". En Water International. Año 37File Attachments, № 2, pp. 131-146.

Rendic VÉlIZ, Dinko Tomislav (2009): Derechos de Agua y Pueblos Indígenas [Water Rights and Indigenous Peoples], Editorial Librotecnia.

Ríos BreHM y Quiroz (1995): "The Market For Water Rights In Chile" (World Bank Technical Paper Number 285, Washington, and Accessed 9 October 2017). Http://Documents.Worldbank.Org/Curated/En/254241468769894995/Pdf/Multi-Page.Pdf.

Rojas Calderón, C. (2010): La distribución de las aguas en el Derecho Chileno. La Actividad Desarrollada por las Juntas de Vigilancia. Tesis Doctoral. Pontificia Universidad Católica de Chile.

Rojas CALDERÓn, C. (2014): "Autogestión y autorregulación regulada de las aguas: organizaciones de usuario de aguas (OUA) y juntas de vigilancia de ríos". En lus et Praxis. Año 20, N 1, Págs. 123-162.

RuRu, J. (2013): "Indigenous Restitution In Settling Water Claims: The Developing Cultural And Commercial Redress Opportunities In Aotearoa, New Zealand". En Pacific Rim Law \& Policy Journal. S.D. No 2, 311 pp.

Stone, C. D. (2010): Should Trees Have Standing? Law, Morality, and The Environment. S.L., New York: Oxford University Press, C2010.

TeCKLIN, D.; BAUer C. y Prieto M. (2011): Making Environmental Law for The Market: The

Vergara Blanco, Alejandro (1998): Derecho de Aguas [Water Law] (Editorial Jurídica de Chile)

Vergara Blanco, Alejandro (2014): "Autogobierno en la gestión de las aguas en Chile". Crisis del agua en el Perú. $195 \mathrm{pp}$.

Waitangi Tribunal (1999): Whanganui River Report (Wai 167).

Waitangi Tribunal (2012): National Fresh Water and Geothermal Resources Inquiry, Stage One Report (Wai 2357, Wai 2358).

Warnock, C. y Baker-Galloway M. (2014): Focus on Resource Management Law. North Ryde, N.S.W. Cch Australia, 2014.

YANEZ, Nancy y Molina, Raul (2011): Las Aguas Indigenas En Chile [Indigenous Waters in Chile] (Lom Ediciones).

\section{Normativa citada}

Código de Aguas 1981 (Chile)

Constitución de la República del Ecuador 2008 (Ecuador)

Constitución Política de la República de Chile 1980 (Chile)

Constitución Política del Estado 2009 (Bolivia)

Ley de Derechos de la Madre Tierra (Ley 071) 2010 (Bolivia)

Natural Resources Management Act 2004 (SA, Australia)

Resource Management Act 1993 (NZ)

Rights in Water and Irrigation Act 1914 (WA, Australia) 
Te Awa Tupua (Whanganui River Claims Settlement) Act 2017 (NZ)

Te Urewera Act 2014 (NZ)

Waikato-Tainui Raupatu Claims (Waikato River) Settlement Act 2010 (NZ)

Water Act (NT, Australia)

Water Act 1989 (Vic, Australia)

Water Act 2000 (Qld, Australia)

Water Management Act 1999 (Tas, Australia)

Water Management Act 2000 (NSW, Australia)

\section{Jurisprudencia citada}

Centro de Estudios para la Justicia Social 'Tierra Digna' y otros con el Presidente de la Republica y otros: 10 noviembre 2016, Corte Constitucional, Sala Sexta de Revisión (No T-5.016.242) (Colombia).

Junta de Vigilancia de la Tercera Sección del Río Mapocho con Aguas Andinas S.A.: 27 abril 2017, Segundo Tribunal Ambiental (D-24-2016) (Chile).

Mohd. Salim con State of Uttarakhand \& others: 20 March 2017, High Court of Uttarakhand (WPPIL 126/2014) (India). 